\title{
On the gravitational seesaw in higher-derivative gravity
}

\author{
Antonio Accioly ${ }^{1, \mathrm{a}}$, Breno L. Giacchini ${ }^{1, \mathrm{~b}}$, Ilya L. Shapiro ${ }^{2,3,4, \mathrm{c}}$ \\ ${ }^{1}$ Centro Brasileiro de Pesquisas Físicas, Rua Dr. Xavier Sigaud 150, Urca, Rio de Janeiro, RJ 22290-180, Brazil \\ 2 Departamento de Física, ICE, Universidade Federal de Juiz de Fora, Campus Universitário, Juiz de Fora, MG 36036-330, Brazil \\ ${ }^{3}$ Tomsk State Pedagogical University, Tomsk, Russia \\ 4 Tomsk State University, Tomsk, Russia
}

Received: 14 June 2017 / Accepted: 27 July 2017 / Published online: 12 August 2017

(C) The Author(s) 2017. This article is an open access publication

\begin{abstract}
Local gravitational theories with more than four derivatives are superrenormalizable. They also may be unitary in the Lee-Wick sense. Thus it is relevant to study the low-energy properties of these theories, especially to identify observables which might be useful for experimental detection of higher derivatives. Using an analogy with the neutrino physics, we explore the possibility of a gravitational seesaw mechanism in which several dimensional parameters of the same order of magnitude produce a hierarchy in the masses of propagating particles. Such a mechanism could make a relatively light degree of freedom detectable in low-energy laboratory and astrophysical observations, such as torsionbalance experiments and the bending of light. We demonstrate that such a seesaw mechanism in the six- and morederivative theories is unable to reduce the lightest mass more than in the simplest four-derivative model. Adding more derivatives to the four-derivative action of gravity makes heavier masses even greater, while the lightest massive ghost is not strongly affected. This fact is favorable for protecting the theory from instabilities but makes the experimental detection of higher derivatives more difficult.
\end{abstract}

\section{Introduction}

The role of higher derivatives in quantum and classical gravity theories is important, complicated and ambiguous. On the one hand it is well known that semiclassical [1] and quantum [2] gravity can be formulated as renormalizable theories only with the four-derivative terms in the action (see [3,4] for an introduction and [5] for a recent review). On the other hand, by adding higher-derivative terms to the Einstein-Hilbert action one introduces massive unphysical ghosts, related instabilities and (in the quantum gravity case)

\footnotetext{
a e-mail: accioly@cbpf.br

be-mail: breno@cbpf.br

c e-mail: shapiro@fisica.ufjf.br
}

a non-unitary $S$-matrix. Recently it was shown that in a theory with six or more derivatives all massive poles can be complex and the $S$-matrix becomes unitary in the Lee-Wick sense [6].

Let us remember that higher derivatives also emerge in the gravitational effective action in string theory. The corresponding terms are removed by means of the Zwiebach reparametrization of the background metric in target space [7-9]. However, this procedure is ambiguous since the noghost condition does not fix many terms in the higherderivative sector [10]. Furthermore, another source of ambiguity is that the problem may be solved not only by completely removing all potentially dangerous terms but also by reducing the effective action to a ghost-free non-local form [11].

It is important to note that in both of these approaches the removal of massive ghosts requires an absolutely precise finetuning of the action. Nevertheless, any small violation here should lead to destructive instabilities and, moreover, these instabilities are even stronger for smaller violations [12]. This means that the ghost-killing procedure in string theory [79] (or [11]) demands an absolutely precise fine-tuning of infinitely many parameters. On the other hand, violations of the fine-tuning cannot be avoided if the loop contributions are taken into account [13] in the effective field theory framework (see, e.g., [14]). The most reasonable position all in all is to assume the existence of higher derivatives and try to understand why they do not produce a total destruction of the classical gravitational solutions $[15,16] .{ }^{1}$

Keeping the string theory in mind, it can be assumed that the action of the theory has only one fundamental dimen-

\footnotetext{
1 The results of $[15,16]$ are coherent with previous works on the stability of the de Sitter space in fourth order gravity, which was first considered in [17]. A more detailed analysis of cosmic perturbations in four-derivative gravity, with qualitatively similar conclusions, was given in a recent work [18].
} 
sional parameter, the Planck mass. All dimensionless coefficients are supposed to be of order one. Hence all phenomena which occur at sub-Planckian energies may be considered as low-energy ones. Then, assuming that there is no fine-tuning and that the higher-derivative terms are there, the natural questions are: (i) Do we have a chance to see the effect of higher-derivative terms at low energies? (ii) Is the IR gravitational physics protected from the ghosts if the dimensional parameters are all related to the Planck mass? These questions are particularly relevant because already at the semiclassical level the loop corrections produce non-local form factors in the quadratic curvature terms.

At low energies it is, for the sake of simplicity, natural to assume a truncation of the infinite series in the d'Alembert operator, leading to an effective polynomial theory of the type [19]

$$
\begin{aligned}
S= & \frac{1}{16 \pi G} \int \mathrm{d}^{4} x \sqrt{-g}(R-2 \Lambda) \\
& +\int \mathrm{d}^{4} x \sqrt{-g}\left\{c_{1} R_{\mu \nu \alpha \beta}^{2}+c_{2} R_{\mu \nu}^{2}+c_{3} R^{2}\right. \\
& +d_{1} R_{\mu \nu \alpha \beta} \square R^{\mu \nu \alpha \beta}+d_{2} R_{\mu \nu} \square R^{\mu \nu}+d_{3} R \square R \\
& +d_{4} R^{3}+d_{5} R R^{\mu \nu} R_{\mu \nu}+\cdots+f_{1} R_{\mu \nu \alpha \beta} \square^{k} R^{\mu \nu \alpha \beta} \\
& \left.+f_{2} R_{\mu \nu} \square^{k} R^{\mu \nu}+f_{3} R \square^{k} R+\cdots+f_{\ldots} R_{\ldots}^{k+2}\right\},
\end{aligned}
$$

where we have used the same sign conventions as in [20].

In what follows we will be interested in the modified Newtonian potential and the bending of light by a weak gravitational field. In this spirit the cosmological constant term and those terms which are third- or higher-order in curvature can be disregarded. Furthermore, for the sake of simplicity, we start the analysis from the $k=1$ case. As a consequence the relevant part of the action can be cast into the form

$$
\begin{aligned}
S= & S_{\text {grav }}+\int \mathrm{d}^{4} x \sqrt{-g} \mathcal{L}_{m}, \\
S_{\text {grav }}= & \int \mathrm{d}^{4} x \sqrt{-g}\left\{\frac{2}{\kappa^{2}} R+\frac{\alpha}{2} R^{2}+\frac{\beta}{2} R_{\mu \nu}^{2}\right. \\
& \left.+\frac{A}{2} R \square R+\frac{B}{2} R_{\mu \nu} \square R^{\mu \nu}\right\},
\end{aligned}
$$

where an additional matter action was introduced; besides, the notations were adjusted for the sake of consequent calculations. Here $\alpha, \beta, A$ and $B$ are free parameters, where the first two are dimensionless while $A$ and $B$ have the dimension of (mass $)^{-2}$. In the following part we will refer to the quantities $|B|^{-1 / 2}$ and $|A|^{-1 / 2}$ as the massive parameters of the action. The notation $\kappa^{2} / 2=16 \pi G=M_{P}^{-2}$ is conventional in the quantum gravity literature.

As we have mentioned above, in string theory all massive parameters are constructed from the single dimensional parameter $\alpha^{\prime}$, and hence all masses in the action are supposed to have the same (typically Planck) order of magnitude. However, our experience with the seesaw mechanism in neutrino physics shows that this does not rule out a situation where several huge massive parameters combine into one particle of light mass, with the other masses becoming even greater. In our case the quantities in the action must satisfy $A^{-1}, B^{-1}, \kappa^{-2} \sim M_{P}^{2}$. In the present work we discuss the possibility of a seesaw-like mechanism. As we show, in the gravitational case one can have a parameter $B^{-1}$ much smaller than $M_{P}^{2}$ and still have an associate mass of the order of $M_{P}$. This scenario can be achieved by reducing the lighter mass of the tensor excitation, which is the well-known ghost mode.

The paper is organized as follows. In Sect. 2 we discuss the new gravitational seesaw mechanism in the theory with more than four derivatives, such as in (3). In Sect. 3 the results of the previous section are extended to the case in which the propagator has complex poles. Possible observational effects caused by a light particle are briefly commented in Sect. 4. We remark that the main focus of this communication is on the seesaw mechanism, while the detailed discussion concerning phenomenological aspects of the theory with six or more derivatives of the metric will be given in the parallel work [21], devoted to the modified Newtonian potential and the bending of light. Finally, in Sect. 5 we draw our conclusions.

\section{Gravitational seesaw in higher-derivative theories}

The conventional point of view is that higher derivatives are not observable at low energies because of the Planck suppression. In order to have the Planck suppression in fourderivative gravity the coefficients of the higher-order terms have to be of order one or at least not too many orders of magnitude greater. However, what is correct as far as the four-derivative model is concerned is not necessary right for theories exhibiting six derivatives or more. Since there are several massive parameters a specific seesaw-like mechanism can be imagined, which enables two (or more) largemass parameters to combine in such a way that they produce a much smaller physical mass. Let us examine in this respect the theory (3).

In the weak-field limit, i.e. $g_{\mu \nu}=\eta_{\mu \nu}+\kappa h_{\mu \nu}$ and $\left|\kappa h_{\mu \nu}\right| \ll 1$, the linearized field equations can be cast into the form

$$
\begin{aligned}
& \left(\frac{2}{\kappa^{2}}-\frac{\beta}{2} \square-\frac{B}{2} \square^{2}\right)\left(R_{\mu \nu}-\frac{1}{2} \eta_{\mu \nu} R\right) \\
& -\left(\alpha+\frac{\beta}{2}+A \square+\frac{B}{2} \square\right)\left(\eta_{\mu \nu} \square R-\partial_{\mu} \partial_{\nu} R\right)=-\frac{T_{\mu \nu}}{2} .
\end{aligned}
$$

It is possible to show that, introducing a suitable gauge condition, the weak gravitational field generated by a static point- 
like mass, $T_{\mu \nu}(\mathbf{r})=M \eta_{\mu 0} \eta_{\nu 0} \delta^{(3)}(\mathbf{r})$, has non-zero components given by (more detailed and general results are found in $[22,23])$

$h_{00}=\frac{M \kappa}{16 \pi}\left(-\frac{1}{r}+\frac{4}{3} F_{2}-\frac{1}{3} F_{0}\right)$,

$h_{11}=h_{22}=h_{33}=\frac{M \kappa}{16 \pi}\left(-\frac{1}{r}+\frac{2}{3} F_{2}+\frac{1}{3} F_{0}\right)$,

where

$F_{k}=\frac{\mu_{k+}^{2}}{\mu_{k+}^{2}-\mu_{k-}^{2}} \frac{\mathrm{e}^{-\mu_{k-} r}}{r}+\frac{\mu_{k-}^{2}}{\mu_{k-}^{2}-\mu_{k+}^{2}} \frac{\mathrm{e}^{-\mu_{k+} r}}{r}$.

Here $k=0,2$ labels the spin of the particles, whose masses are defined by the positions of the poles of the propagator,

$\mu_{2 \pm}^{2}=\frac{\beta \pm \sqrt{\beta^{2}+\frac{16}{\kappa^{2}} B}}{2 B}, \quad \mu_{0 \pm}^{2}=\frac{\sigma_{1} \pm \sqrt{\sigma_{1}^{2}-\frac{8 \sigma_{2}}{\kappa^{2}}}}{2 \sigma_{2}}$,

with $\sigma_{1} \equiv 3 \alpha+\beta$ and $\sigma_{2} \equiv 3 A+B$. In the sixth-order gravity massive particles occur in dependent pairs with the same spin. The masses (6) are real and non-degenerate provided that

$\beta, B<0, \quad \sigma_{1}, \sigma_{2}>0$ and

$\beta^{2}+\frac{16 B}{\kappa^{2}}>0, \quad \sigma_{1}^{2}-\frac{8 \sigma_{2}}{\kappa^{2}}>0$.

Indeed, the quantities $\mu_{0 \pm}$ and $\mu_{2 \pm}$ could be complex and still yield a real solution to the equations of motion (4) and thus physically admissible results, e.g., through a real effective potential [21,23]. Here, however, we restrict the analysis to the case of real poles, while the scenario with complex poles is explored in the following section.

Let us start the discussion of the mass relations in the sixderivative theory from considering the tensor sector. According to Eq. (6) for the case of real poles with $\mu_{2+}^{2}<\mu_{2-}^{2}$ the lighter massive excitation is a ghost and the other is a healthy tensor field [19]. Using Eqs. (6) and (7) it is easy to show the existence of a relation between $\beta$ and $B$, namely $16|B| \ll \kappa^{2} \beta^{2}$, in the special case when one of the masses is much smaller than the other,

$\mu_{2+}^{2} \ll \mu_{2-}^{2}$.

In the theory where this condition is satisfied, the masses $\mu_{2 \pm}$ can be approximated by

$\mu_{2+}^{2} \approx \frac{4}{\kappa^{2}|\beta|} \ll \mu_{2-}^{2} \approx \frac{\beta}{B}$.

As in the original neutrino's seesaw mechanism one of the masses roughly depends on only one parameter while the other depends on both. Moreover, this relation occurs in such a manner that if the lighter mass is reduced the larger mass is augmented. A remarkable difference with respect to the neutrino's mechanism is that while in the neutrino case it works to make the lightest mass even lighter, in the gravitational model the effect is to turn the largest mass even larger according to Eq. (9). This happens due to the presence of the parameter $B$ in the denominator of Eq. (6), making the lightest mass depend only on $\beta$, while the largest one depends on both parameters.

In this vein, there are two possible ways of having $\mu_{2-}$ of the order of the Planck mass: to have a small $|B|$ or a larger $|\beta|$. The first choice is the standard one, since it prescribes that $\beta \sim 1$ and $B \sim M_{P}^{-2}$ so as to have all the masses to the order of $M_{P}$. The second possibility, which relies on the seesaw mechanism, allows one to have $|B| \gg M_{P}^{-2}$ and still have $\mu_{2-} \sim M_{P}$. Of course, having a large $|B|$ still yielding one large mass can only be achieved by means of the ghost mass reduction through a parameter $\beta \gg 1$. The final result, as can be seen from Eq. (9), is that the existence of a much lighter mass of the first (ghost) state depends only on the second- and fourth-derivative terms while the six-derivative term does not affect the presence of much lighter mass. Mutatis mutandis these arguments also apply to the scalar modes. By the end of the day, the six-derivative terms are not capable to produce an efficient seesaw mechanism working like in the case of the neutrino mass.

A general argument in favor of the non-possibility of the strong seesaw mechanism for even higher-order gravity theories, i.e. eight and more, can be presented. For instance, consider the action (1) with $k=2$ which means the eightderivative theory. One can write the equation for the massive poles in the propagator in the form

$\frac{1}{m_{0}^{4}} k^{6}-\frac{3}{m_{1}^{2}} k^{4}+3 \beta k^{2}-m_{2}^{2}=0$.

Here $m_{0,1,2}$ are positive massive parameters coming from the action. In string theory it is assumed that they are all of the same order of magnitude, say

$m_{0}^{2} \sim m_{1}^{2} \sim m_{2}^{2} \sim M_{P}^{2}$.

Assuming this is the case (10) can be rewritten in the more simple form

$k^{6}-\frac{3 m_{0}^{4}}{m_{1}^{2}} k^{4}+3 \beta m_{0}^{4} k^{2}-m_{0}^{4} m_{2}^{2}=0$.

The roots of this equation are defined by the Cardano formula and can be real or complex. Consider the particular case of real positive roots which satisfy the hierarchy $\mu_{1}^{2} \ll \mu_{2}^{2} \sim$ $\mu_{3}^{2}$. Then the equation becomes

$$
\begin{aligned}
k^{6} & -\left(\mu_{1}^{2}+\mu_{2}^{2}+\mu_{3}^{2}\right) k^{4}+\left(\mu_{1}^{2} \mu_{2}^{2}+\mu_{1}^{2} \mu_{3}^{2}+\mu_{2}^{2} \mu_{3}^{2}\right) k^{2} \\
& -\mu_{1}^{2} \mu_{2}^{2} \mu_{3}^{2}=0 .
\end{aligned}
$$

Using the hierarchy $\mu_{1}^{2} \ll \mu_{2}^{2} \sim \mu_{3}^{2}$, the last equation boils down to

$k^{6}-\left(\mu_{2}^{2}+\mu_{3}^{2}\right) k^{4}+\mu_{2}^{2} \mu_{3}^{2} k^{2}-\mu_{1}^{2} \mu_{2}^{2} \mu_{3}^{2}=0$. 
It is easy to see that there is a contradiction between Eq. (12) with (11) and Eq. (14). According to (12) we have

$$
\begin{aligned}
& \frac{3 m_{0}^{4}}{m_{1}^{2}} \sim M_{P}^{2}, \quad 3 \beta m_{0}^{4} \sim M_{P}^{4} \\
& \text { and } \quad m_{0}^{4} m_{2}^{2} \sim M_{P}^{6} .
\end{aligned}
$$

However, this does not fit Eq. (14), because this requires

$\mu_{2}^{2}+\mu_{3}^{2} \sim M_{P}^{2}, \quad \mu_{2}^{2} \mu_{3}^{2} \sim M_{P}^{4}$,

but $\mu_{1}^{2} \mu_{2}^{2} \mu_{3}^{2} \ll M_{P}^{6}$.

This consideration can easily be extended to the higher number of derivatives. The result will always be the same. We leave it as an exercise to the interested reader. It is also worth stressing that this general reasoning applies to both the tensor and the scalar sectors of the model. Finally, the mechanism that actually may take place in higher-derivative gravity can be called "weak seesaw". A larger mass can become even larger while a smaller one does not become smaller without using unnatural values for the dimensionless parameters of the action.

The main conclusion is that the real poles of the propagator cannot provide a much smaller mass of the lightest ghost constructed from the coefficients which are all of the Planck order of magnitude. Is this bad or not from the physics side? We know that the presence of ghost means potential instability but, in the case of gravity, the situation may be different $[15,16]$, for instance because of the singular nature of nonpolynomial theory which escapes the Ostrogradsky instability [12]. Since a consistent theory of quantum or semiclassical gravity without higher derivatives looks impossible, the general situation with stability looks unclear and it makes sense to assume that ghosts exist but for some reason they do not lead to a fast decay of the vacuum and other type of instabilities. The existing explanation for this is related to the huge mass of the ghost [15,16] (not tachyon! - see [24]) which does not permit the creation of a ghost particle from vacuum without generating Planck-order density of gravitons. From this perspective it is important that the mass of the lightest ghost is protected from the seesaw mechanism if even more derivatives are added to the action (1).

The mass of the lightest scalar (healthy) excitation is likewise protected from the seesaw mechanism. Let us note that the successful realization of the Starobinsky inflation model [25] requires a large value of the coefficient of the $R^{2}$ term $[26,27]$. Such a coefficient is reducing the mass of the scalar mode considerably but this happens without strong seesaw mechanism. It remains to see what would be further phenomenological consequences of the light ghost (and/or of a light scalar particle) within the weak seesaw mechanism as in (8). In Sect. 4 we consider an example of this kind.

\section{Seesaw with complex poles}

In the previous section we have dealt only with the case in which the propagator has real poles. Of course, if the quantities $\mu_{i}$ are complex it does not make sense to consider a seesaw-like mechanism meaning a strong hierarchy between those "masses". This idea, however, can be extended keeping in mind the original motivation for considering a gravitational seesaw: to have huge-mass parameters in the action resulting in a small physical particle mass. Hence, we shall define the seesaw mechanism in the case of complex poles as a way of having huge-mass parameters in the action yielding small physical massive parameters, which turn out to be the real and the imaginary parts of $\mu_{i}$.

Instead of starting from the six-derivative gravity example as we proceeded for the case of real poles we shall now go directly to the general proof outlined in the previous section. Since the poles of the propagator are defined as the roots of a polynomial equation such as (10) it follows from the fundamental theorem of algebra that complex poles always occur in conjugate pairs. Let $\mu_{1}=a+i b$ and $\mu_{1^{\prime}}=a-i b$ be one such pair; then, writing the equation for the poles in terms of the roots, as in (13), the coefficient formed by the sum of all the roots (squared) will contain the term $2\left(a^{2}-b^{2}\right)$. The coefficient which involves the products of the roots chosen two by two will have the terms

$$
\begin{aligned}
\mu_{1}^{2} \mu_{1^{\prime}}^{2} & =a^{4}+b^{4}+2 a^{2} b^{2}, \\
\mu_{1}^{2} \mu_{2}^{2}+\mu_{1^{\prime}}^{2} \mu_{2}^{2} & =2\left(a^{2}-b^{2}\right) \mu_{2}^{2},
\end{aligned}
$$

for an arbitrary third root $\mu_{2}^{2}$ and so on.

Of course, all the coefficients will be real since the parameters of the action are also real. In this sense there is not much difference with the case of real poles, apart from the fact that now the relevant quantities are $a^{2}$ and $b^{2}$. The last term, however, formed by the product of all the roots, will contain the term $\mu_{1}^{2} \mu_{1}^{2}$ given by (17). This conversion of the product of all the roots into a sum changes the argument used in the last section to show that the strong seesaw mechanism does not work. In fact, thinking of the eight-derivative model above, it is well possible to have $m_{0}^{4} m_{2}^{2} \sim M_{P}^{6}$ in Eq. (15) at the same time in Eq. (16) we have

$\mu_{1}^{2} \mu_{1^{\prime}}^{2} \mu_{2}^{2}=\left(a^{4}+b^{4}+2 a^{2} b^{2}\right) \mu_{2}^{2} \sim M_{P}^{6}$

with either $a^{2} \ll b^{2} \sim M_{P}^{2}$ or $b^{2} \ll a^{2} \sim M_{P}^{2}$.

This means that it is possible to have a strong hierarchy between the real and imaginary parts of the roots $\mu_{i}-$ with huge-mass parameters in the action. Yet we do not call it a working seesaw mechanism because the physical effects of such a hierarchy does not imply a way out of the Planck suppression. It only means that the largest among the real or the imaginary part is going to dominate the phenomenology and that this quantity is on the order of $M_{P}$. Indeed, if 
$b^{2} \ll a^{2} \sim M_{P}^{2}$, then to most practical purposes it can be assumed that the pair $\left(\mu_{1}, \mu_{1^{\prime}}\right)$ behaves as degenerate modes of mass $a \sim M_{P}$; while if $a^{2} \ll b^{2} \sim M_{P}^{2}$ they behave as a degenerate tachyonic pair.

Moreover, this procedure proves that there is no natural choice of massive parameters in the action which can provide a simultaneous reduction of both real and imaginary parts of the complex "masses" of the theory. In conclusion there is no seesaw-like mechanism efficiently working in the polynomial higher-derivative gravity, even if the propagator contains complex poles.

In order to close this section it is instructive to explicitly work out the aforementioned example of the six-derivative gravity (3). According to (6), the condition for having complex poles in the propagator of the tensor modes reads $\beta^{2} \kappa^{2}+16 B<0$. The "masses" $\mu_{2 \pm}$ can now be written as $\mu_{2 \pm}=a_{2} \mp i b_{2}$ so that

$a_{2}^{2}=\frac{-\beta+\sqrt{\frac{16|B|}{\kappa^{2}}}}{4|B|}, \quad b_{2}^{2}=\frac{\beta+\sqrt{\frac{16|B|}{\kappa^{2}}}}{4|B|}$.

The possible situation can be classified as follows. In case that $16|B|$ is only slightly larger than $\beta^{2} \kappa^{2}$ there is a strong hierarchy between real and imaginary parts and the "masses" $\mu_{2 \pm}$ tend to be approximately equal. In fact, if $\beta<0$ we get $a_{2} \gg b_{2}$ and both excitations behave almost like normal particles of the same mass, while $\beta>0$ yields $a_{2} \ll b_{2}$ and we have two tachyons. If $16|B| \approx \beta^{2} \kappa^{2} \sim M_{P}^{-2}$, then

$\mu_{2 \pm}^{2} \approx-\frac{8}{\beta \kappa^{2}} \sim M_{P}^{2}$

This figure can be reduced only by choosing a huge $|\beta|$ (and simultaneously, in this case, a huge $|B|$ ).

On the other hand, if $16|B| \gg \beta^{2} \kappa^{2}$, there are "masses" with real and imaginary parts of the same order of magnitude. This scenario is a truly complex one; however, since $a_{2} \approx b_{2}$, one can work with a single massive parameter. Therefore the only possibility for a seesaw mechanism would be to reduce this quantity far below the Planck mass. This notwithstanding, we have

$a_{2}^{2} \approx b_{2}^{2} \approx \sqrt{\frac{1}{\kappa^{2}|B|}}=\frac{M_{P}}{\sqrt{2|B|}}$.

Therefore, in order to have $a_{2}^{2}, b_{2}^{2} \ll M_{P}^{2}$, it is necessary to impose $|B| \gg M_{P}^{-2}$. Reducing the parameter $\beta$ cannot diminish the effective mass. The only way of achieving this is by increasing $|B|$ to unnatural values, i.e. by applying the "weak seesaw" condition. It is interesting to notice that, in opposition to the real poles weak seesaw (choosing a huge $|\beta|$, cf. (9)), in the case of complex poles it is a condition on $B$. A similar discussion applies to the complex scalar modes $\mu_{0 \pm}$.

\section{On the physical consequences of gravitational seesaw}

From the general perspective it is interesting to discuss what could be the phenomenological consequences of the much lighter massive ghost. Let us note that these and related subjects are discussed in detail in the context of the general sixderivative model in the parallel paper [21]. Here we present just a brief extract of the results which have a relation to the seesaw mechanism. Let us start from some obvious statements.

The presence of light excitations in the spectrum of the theory would reduce the Planck suppression at both classical and semiclassical cases and would bring the physical relevance of the massive modes to the low-energy domain. This would imply, for example, modifications of Newton's inverse-square force law [21-23] which is measured in torsion-balance experiments $[28,29]$. In the case of complex poles for example, the corrections owed to the higher derivatives assume the form of oscillating terms as was noticed in $[21,30]$. A stimulating discussion on the perspective of detecting oscillations in the gravitational potential can be found in [31]. An important theoretical feature of the higherderivative corrections to the Newton gravitational law is that the relevant contributions come from both tensor and scalar sectors of the theory [2,22].

Another possibility of detecting signatures of higher derivatives is the gravitational light bending. In this case the tensor and scalar excitations play different roles. It was shown (see, e.g., [20]) that the deflection of light in the four-derivative gravity explicitly depends only on the tensor modes of metric perturbations.

It proves interesting to discuss this issue in full detail. Let us start from the simplest case. The linearized versions of general relativity and the $R+R^{2}$ gravity model yield the same equations of motion for photons [32]. On the other hand, the $R+R^{2}$ model is equivalent to the Brans-Dicke theory with a massive degree of freedom. It is possible to show that the light bending alone cannot distinguish between this type of metric-scalar gravity theories and general relativity [32-35]. In these references one can find the discussion concerning the difference with the massless Brans-Dicke theory [36], in which the mass of the massive body (e.g., a star or galaxy) creating the gravitational field must be renormalized.

The reason for the difference between the massive and massless Brans-Dicke theories in the framework of the equivalent $R+R^{2}$ gravity model is easily understood. The need of rescaling Newton's constant $G$ and/or of the measured masses of astronomical bodies is that the Yukawa term in the modified Newtonian potential [2]

$V(r)=-\frac{G M}{r}\left(1+\frac{1}{3} \mathrm{e}^{-\mu_{0} r}\right)$

becomes in the massless or very light mass limit $\mu_{0} \rightarrow 0$ the same as the Newtonian term. Hence, effectively in the 
massless limit $G M$ can be replaced by $4 G M / 3$, as occurs in the massless Brans-Dicke theory.

The presence of a massive scalar mode with $\mu_{0}>0$ also affects the effective quantity $G M$ but only at the range of distances below $1 / \mu_{0}$. For the massive Brans-Dicke theories this was explicitly shown in Ref. [37]. For the properly chosen small $\mu_{0}$ this leads to a mismatch between an effective mass $M$ which can be observed at the astrophysical and laboratory scales [38] and may affect the predictions for the deflection of light. This result can indeed be generalized for the $R+R \square R$ and more general models with even more derivatives. At the same time in the case of higher-derivative gravity models with six or more derivatives such a light scalar with Compton wavelength at the astronomical scale is out of scope of the present work. Therefore we assume much larger masses of the massive gravitational modes.

The introduction of massive parameters in the tensor sector, on the other hand, has a direct influence on the deflection of light. At quantum level it yields scattering cross-sections that depend on the energy of the photon, as was shown, e.g., in Refs. [20,21,39]. This result, however, has little application for the deflection by astronomical bodies [21]. From the classical perspective those tensor modes only play an active role if the reciprocal of their masses are comparable to the light ray impact parameter (see [20] for a specific discussion on light bending in the model with four derivatives and [21] for the one with six derivatives and all possible scenarios for the massive poles). In the case of the polynomial model with real poles in the propagator, this would require a light ghost which could be provided by the (weak) seesaw mechanism. Nonetheless, light deflection by the Sun cannot yield better observational constraints on such masses than torsionbalance experiments at laboratory scales [21,29].

To conclude the discussion on light deflection, if the seesaw mechanism works in at least one of the massive sectors, the effects of such light masses are more likely to be observed in the modified Newtonian potential than on the bending of light in the Solar System.

Last but not least, a light ghost could affect the cosmology and especially the stability of classical solutions with respect to tensor perturbations. For the four-derivative models this issue was discussed in [15-18]. The absence of the effectively working seesaw mechanism shows that the Planck protection which was discussed in $[15,16,40]$ is working the same way in the six-derivative (and higher-derivative) gravity.

\section{Conclusions and discussions}

We have described a qualitatively new gravitational seesaw mechanism which might be possible in the higher-derivative gravity models with the number of derivatives $\geq 6$. These theories are characterized by a discrete spectrum of "masses" which may be real or complex.

If the dimensional parameters of the action have Planck order of magnitude, they could combine, in principle, in such a way that one of the masses is still on the order of $M_{P}$ while another is many orders of magnitude smaller. As we have seen above such a strong gravitational seesaw is not possible. An essential reduction of the mass of the lightest particle can be achieved only by adjusting the four-derivative term in the action. Adding the six-derivative terms does not modify the situation in this part.

A strong reduction of the mass of the lightest tensor ghost can be achieved by taking a huge value of the dimensionless parameter $\beta$, exactly like in the four-derivative gravity. This situation is qualitatively similar to the one in the extradimensional theories. The difference is that here the reduction of Planck suppression occurs due to the choice of $\beta$ and not because of the incomplete compactification of some extra dimensions.

In the more realistic case of complex poles it is still possible to extend the notion of a seesaw mechanism, which should now be understood in the sense that the real massive parameters that appear in the physical quantities of the theory are much below the Planck scale. Nevertheless, we showed that this could only be achieved by simultaneously reducing both real and imaginary parts of one of the poles of the propagator, which only happens with small massive parameters in the action. Thus, only the weak seesaw is possible even in the model with complex poles. Further investigations on general higher-derivative models with complex poles are carried out in [23].

Finally, we briefly mentioned some phenomenological aspects which could be investigated in the presence of the (weak) seesaw mechanism; namely, the modified Newtonian potential and the bending of light. Further consideration on these issues can be found in [21] whose main focus is on lowenergy aspects of the general theory with six derivatives.

Taking into account the possibility to have a continuous mass spectrum of the models such as [11], the main conclusions that can be drawn at the moment are as follows:

(i) A strong gravitational seesaw does not work in the same way like in neutrino physics. The lightest ghost mass cannot be reduced by tuning the parameters of the higher-derivative action (1), except the dimensionless parameter $\beta$.

(ii) Since huge values of the dimensionless parameters $\alpha$ and $\beta$ cannot be completely ruled out theoretically it is important to derive the corresponding upper limits from experimental and observational sides. In the present work we made some steps in this direction.

(iii) Our results indicate the importance of developing experimental facilities for higher precision tests of the 
inverse-square force law since the detection of such an effect could provide a useful information on higher derivatives in gravity. In particular, it looks relevant to explore the possibility of an oscillating behavior of the gravitational potential which is typical for the complex poles [21].

(iv) From the theoretical side the generally negative result for the gravitational seesaw mechanism is relevant for the alternative approach for dealing with the ghost problem, suggested in [41-43]. In this case the higherderivative terms and the corresponding loop contributions are regarded as small corrections to the EinsteinHilbert action. This type of approximation may work only if all the ghost-like states belong to the far UV compared to the scale of the gravitational phenomena. Keeping this in mind this is certainly an efficient ad hoc scheme, not taking into account the price to pay for it, which is that the Planck-scale energies cannot be dealt with and that, for instance, the Starobinsky model of inflation [25,26] must be forbidden [44]. Anyway, our results are very relevant for this approach, since it can be extended to theories with more than four derivatives. As far as the reduction of the effective gravitational ghosts masses does not occur without unnatural choice of dimensionless parameters, the mentioned scheme can be used in the more general kind of theories.

Acknowledgements B.L.G. and I.Sh. are very grateful to Prof. Starobinsky for critical observations and useful discussions, which helped us to improve the paper. A.A. is very indebted to CNPq and FAPERJ, while B.L.G. is thankful to CNPq for supporting his Ph.D. project. I.Sh. is grateful to CNPq, FAPEMIG and ICTP for partial support of his work. Part of it was done during his visit to ICTP and it is a pleasure to thank the high-energy group for warm hospitality.

Open Access This article is distributed under the terms of the Creative Commons Attribution 4.0 International License (http://creativecomm ons.org/licenses/by/4.0/), which permits unrestricted use, distribution, and reproduction in any medium, provided you give appropriate credit to the original author(s) and the source, provide a link to the Creative Commons license, and indicate if changes were made. Funded by $\mathrm{SCOAP}^{3}$.

\section{References}

1. R. Utiyama, B.S. DeWitt, J. Math. Phys. 3, 608 (1962)

2. K. Stelle, Phys. Rev. D 16, 953 (1977)

3. N.D. Birell, P.C.W. Davies, Quantum Fields in Curved Space (Cambridge University Press, Cambridge, 1982)

4. I.L. Buchbinder, S.D. Odintsov, I.L. Shapiro, Effective Action in Quantum Gravity (IOP Publishing, Bristol, 1992)

5. I.L. Shapiro, Class. Quantum Grav. 25, 103001 (2008). arXiv:0801.0216

6. L. Modesto, I.L. Shapiro, Phys. Lett. B 755, 279 (2016). arXiv: 1512.07600

7. B. Zwiebach, Phys. Lett. B 156, 315 (1985)

8. S. Deser, A.N. Redlich, Phys. Lett. B 176, 350 (1986)
9. A.A. Tseytlin, Phys. Lett. B 176, 92 (1986)

10. A.L. Maroto, I.L. Shapiro, Phys. Lett. B 414, 34 (1997)

11. A.A. Tseytlin, Phys. Lett. B 363, 223 (1995). arXiv:hep-th/9509050

12. R.P. Woodard, Lect. Notes Phys. 720, 403 (2007). arXiv:astro-ph/0601672

13. I.L. Shapiro, Phys. Lett. B 744, 67 (2015). arXiv:1502.00106

14. C.P. Burgess, M. Williams, JHEP 1408, 074 (2014). arXiv:1404.2236 [gr-qc]

15. J.C. Fabris, A.M. Pelinson, F. de O. Salles, I.L. Shapiro, JCAP 02, 019 (2012). arXiv: 1112.5202

16. F. de O. Salles, I.L. Shapiro, Phys. Rev. D 89, 084054 (2014). arXiv: 1401.4583

17. V. Müller, H.-J. Schmidt, A.A. Starobinsky, Phys. Lett. B 202, 198 (1988)

18. M.M. Ivanov, A.A. Tokareva, JCAP 1612, 018 (2016). arXiv: 1610.05330

19. M. Asorey, J.L. López, I.L. Shapiro, Int. J. Mod. Phys. A 12, 5711 (1997). arXiv:hep-th/9610006

20. A. Accioly, J. Helayël-Neto, B. Giacchini, W. Herdy, Phys. Rev. D 91, 125009 (2015). arXiv:1506.00270

21. A. Accioly, B.L. Giacchini, I.L. Shapiro, Low-energy effects in a higher-derivative gravity model with real and complex massive poles. arXiv: 1610.05260

22. L. Modesto, T. de Paula Netto, I.L. Shapiro, JHEP 1504, 098 (2015). arXiv:1412.0740

23. B.L. Giacchini, Phys. Lett. B 766, 306 (2017). arXiv:1609.05432

24. G. Cusin, F. de O. Salles, I.L. Shapiro, Phys. Rev. D 93, 044039 (2016). arXiv: 1503.08059

25. A.A. Starobinsky, Phys. Lett. B 91, 99 (1980)

26. A.A. Starobinsky, Sov. Astron. Lett. 9, 302 (1983)

27. S.V. Ketov, A.A. Starobinsky, JCAP 1208, 022 (2012). arXiv: 1203.0805

28. D.J. Kapner et al., Phys. Rev. Lett. 98, 021101 (2007)

29. B.L. Giacchini, Experimental limits on the free parameters of higher-derivative gravity. In: M. Bianchi, R.T. Jantzen, R. Ruffini (Eds.), Proceedings of the 14th Marcel Grossman Meeting on General Relativity. World Scientific, Singapore (2017). arXiv:1612.01823 (in press)

30. L. Modesto, Nucl. Phys. B 909, 584 (2016)

31. L. Perivolaropoulos, Phys. Rev. D 95, 084050 (2017). arXiv: 1611.07293

32. A. Accioly, A.D. Azeredo, E.C. de Rey Neto, H. Mukai, Braz. J. Phys. 28, 2 (1998)

33. M. Lubini, C. Tortora, J. Näf, Ph. Jetzer, S. Capozziello, Eur. Phys. J. C 71, 1834 (2011). arXiv:1104.2851

34. C.P.L. Berry, J.R. Gair, Phys. Rev. D 83, 104022 (2011). (erratum Phys. Rev. D 85, 089906, 2012, arXiv:1104.0819)

35. S. Capozziello, A. Stabile, A. Troisi, Phys. Lett. B 686, 79 (2010). arXiv: 1002.1364

36. C.M. Will, Theory and Experiment in Gravitational Physics, 2nd edn. (Cambridge University Press, Cambridge, 1993)

37. J. Alsing, E. Berti, C.M. Will, H. Zaglauer, Phys. Rev. D 85, 064041 (2012)

38. B. Boisseau, G. Esposito-Farese, D. Polarski, A.A. Starobinsky, Phys. Rev. Lett. 85, 2236 (2000). arXiv:gr-qc/0001066

39. R.R. Caldwell, D. Grin, Phys. Rev. Lett. 100, 031301 (2008)

40. I.L. Shapiro, A.M. Pelinson, F. de O. Salles, Mod. Phys. Lett. A 29, 1430034 (2014). arXiv:1410.2581

41. J.Z. Simon, Phys. Rev. D 41, 3720 (1990)

42. J.Z. Simon, Phys. Rev. D 43, 3308 (1991)

43. L. Parker, J.Z. Simon, Phys. Rev. D 47, 1339 (1993). arXiv:gr-qc/9211002

44. J.Z. Simon, Phys. Rev. D 45, 1953 (1992) 